\title{
TWO-DIMENSIONAL JET AIMED VERTICALLY UPWARDS
}

\author{
JEAN-MARC VANDEN-BROECK ${ }^{\prime}$
}

(Received 31 December 1990; revised 1 July 1991)

\begin{abstract}
A steady two-dimensional jet of an inviscid incompressible fluid rising and falling under gravity is considered. The jet is aimed vertically upwards and the flow is assumed to be bounded entirely by two free surfaces. The problem is solved numerically by finite differences. Accurate results for the free surface profiles are presented.
\end{abstract}

\section{Introduction}

In a recent paper, Dias and Vanden-Broeck [1] calculated numerically twodimensional potential free surface flows of an inviscid incompressible fluid emerging from a nozzle and falling under gravity. The nozzle was aimed at an angle $\beta$ above the horizontal. They found that there are flows for which the flow falls down along the underside of the nozzle (see Figure 1a), flows which split into two sheets (see Figure $1 \mathrm{~b}$ ) and flows with two jets each with two free surfaces (see Figure 1c). Their solutions are determined by the angle $\beta$ and the Froude number

$$
F=\frac{U}{(g H)^{1 / 2}} .
$$

Here $g$ is the acceleration due to gravity, $L$ the width of the nozzle and $U$ the velocity far inside the nozzle.

Dias and Vanden-Broeck [1] showed that for each value of $\beta$, there is a critical value $F(\beta)$ of the Froude number. When $F<F(\beta)$, solutions of the type of Figure 1a occur. When $F>F(\beta)$, solutions of the type of Figure $1 \mathrm{~b}$ occur. In addition they calculated solutions of the type of Figure

\footnotetext{
'Department of Mathematics and Center for the Mathematical Sciences, University of WisconsinMadison, Madison, WI 53705 U.S.A.

(C) Copyright Australian Mathematical Society 1993, Serial-fee code 0334-2700/93
} 

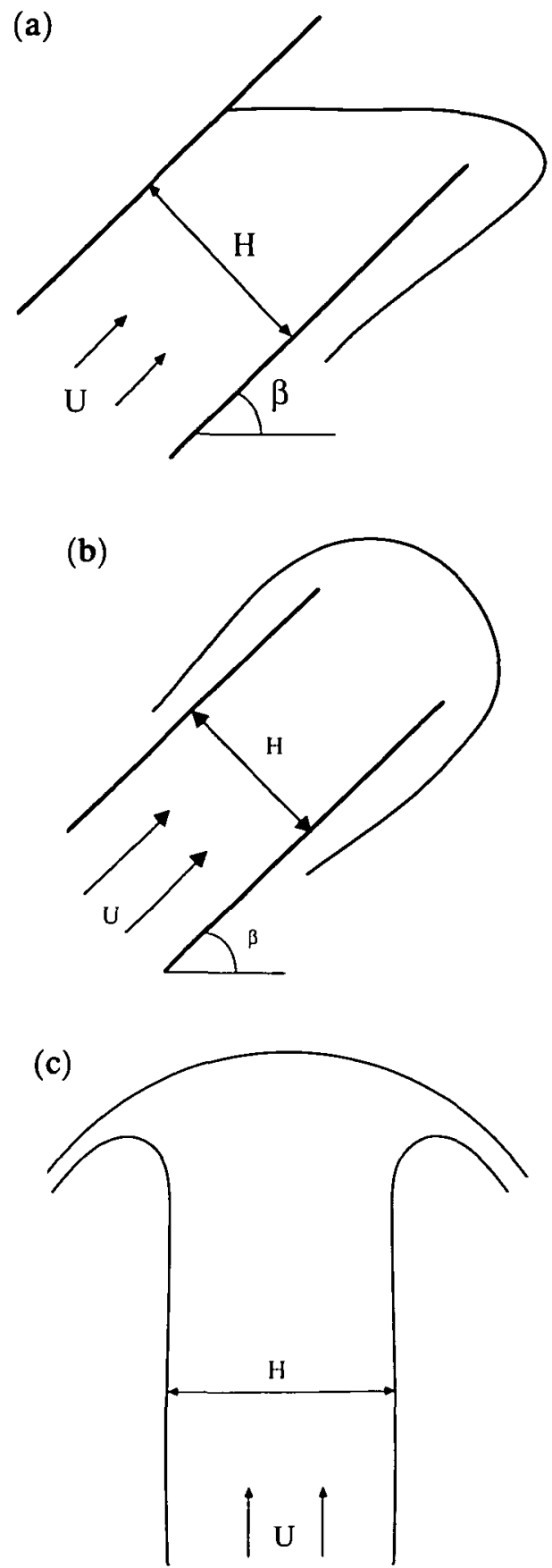

FIGURE 1. Sketch of three different configurations for the flow emerging from a nozzle and falling under gravity. 


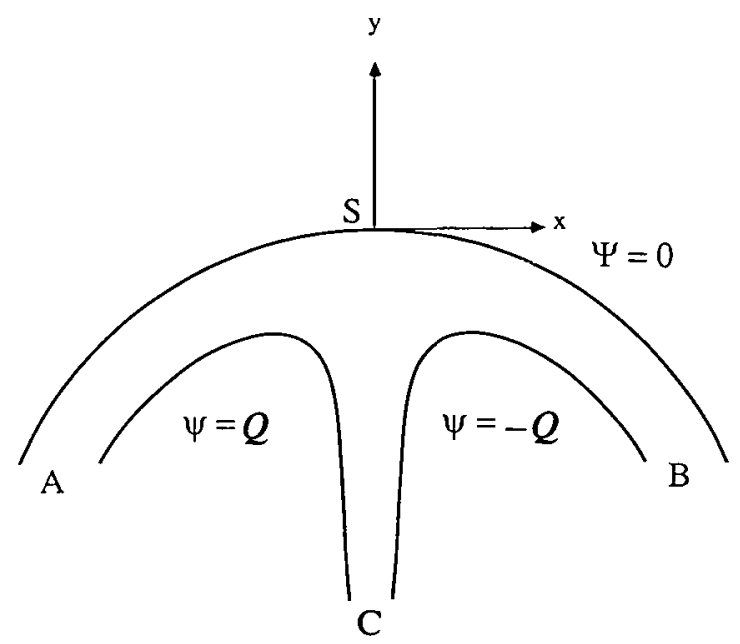

FIGURE 2. Sketch of the free surface profiles of a two-dimensional jet aimed vertically upwards. The flow is bounded entirely by two free surfaces.

$1 \mathrm{c}$, for $\beta=\frac{\pi}{2}$ and small values of the Froude number. These solutions were obtained by approximating the solutions near the lips of the nozzle by the "weir flows" calculated by Vanden-Broeck and Keller [2].

In this paper we assume that $\beta=\frac{\pi}{2}$ and we calculate the limiting configuration of the flow of Figure $1 \mathrm{c}$ as $F \rightarrow \infty$. The nozzle is then infinitely far away and the flow is bounded entirely by two free streamlines (see Figure 2).

The problem is formulated in Section 2 and the numerical results are presented in Section 3.

\section{Formulation}

Let us consider a steady two-dimensional jet bounded by the upper streamline $A S B$ and the lower streamlines $A C$ and $C B$. The fluid is assumed to be inviscid and incompressible. We choose Cartesian coordinates with the origin at the crest $S$ of the jet and the $y$-axis pointing vertically upwards. Gravity is acting in the negative $y$-direction. The flow is assumed to be symmetric about the $y$-axis.

We introduce the potential function $\phi$ and the stream function $\psi$. Without loss of generality, we choose $\phi=0$ at $x=y=0$ and $\psi=0$ on the upper streamline $A S B$ and on the dividing streamline $C S$. We denote by $Q$ the value of $\psi$ on the lower streamline $A C$. It follows from the symmetry of the problem that $\psi=-Q$ on the streamline $C B$. 


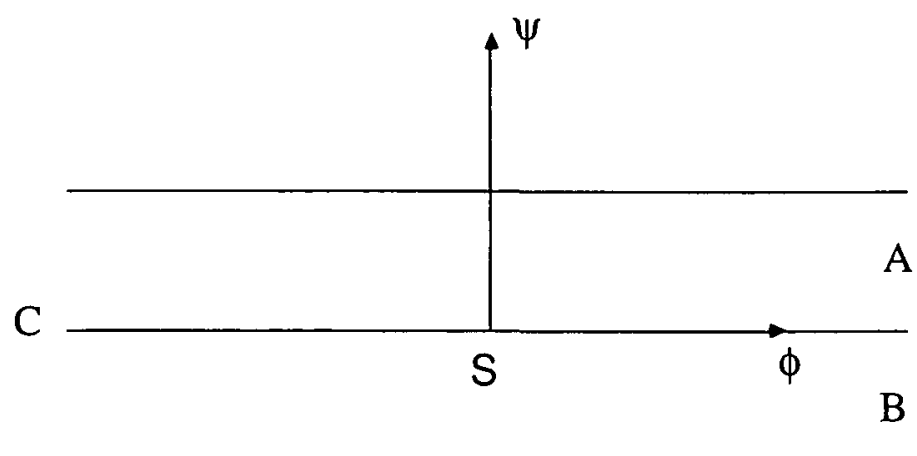

Figure 3. The complex potential plane.

On the streamlines $A S B, A C$ and $C B$, the Bernoulli equation yields

$$
\frac{1}{2}\left(u^{2}+v^{2}\right)+g y=0 .
$$

Here $g$ is the acceleration of gravity and

$$
\begin{gathered}
u=\frac{\partial \phi}{\partial x}=\frac{\partial \psi}{\partial y}, \\
v=\rho \frac{\partial \phi}{\partial y}=-\frac{\partial \psi}{\partial x}
\end{gathered}
$$

are the $x$ - and $y$-components of the velocity, respectively.

We now introduce dimensionless variables by using $\left(Q^{2} / g\right)^{1 / 3}$ as the unit length and $(Q g)^{1 / 3}$ as the unit velocity. In these variables, (2) becomes

$$
u^{2}+v^{2}+2 y=0 \text {. }
$$

The problem is to find $Z=x+i y$ as an analytic function of $f=\phi+i \psi$ in the strip $-1 \leq \psi \leq 1$ of the $f$-plane, with (5) holding on $\psi= \pm 1$ and $\psi=0, \phi>0$. The strip in the $f$-plane is shown in Figure 3. The function $Z(f)$ has a singularity at $f=0$ and is unbounded as $|f| \rightarrow \infty$. The asymptotic behavior as $|f| \rightarrow \infty$ will be described in detail at the end of Section 3. The behavior near $S$ is given by the local analysis of Dagan and Tulin [3]. It shows that the free surface is horizontal at $S$ and that

$$
Z(f) \sim \gamma(f)^{1 / 2} \text { as } f \rightarrow 0 .
$$

Here $\gamma$ is a constant.

We formulate the problem in terms of $y(\phi, \psi)$. Since $y(\phi, \psi)$ is the imaginary part of the analytic function $Z(f)$, it satisfies Laplace equation 
in the strip $-1<\psi<1$. Using the symmetry of the flow, we restrict our attention to the strip $-1<\psi<0$. Therefore we have

$$
\frac{\partial^{2} y}{\partial \phi^{2}}+\frac{\partial^{2} y}{\partial \psi^{2}}=0, \quad-1<\psi<0,-\infty<\phi<+\infty,
$$

together with the symmetry condition

$$
\frac{\partial y}{\partial \psi}=0, \quad \psi=0, \quad-\infty<\phi<0 .
$$

We also rewrite (5) as

$$
\left[\left(\frac{\partial y}{\partial \phi}\right)^{2}+\left(\frac{\partial y}{\partial \psi}\right)^{2}\right]^{-1}+2 y=0 .
$$

The problem is now to find $y(\phi, \psi)$ satisfying (6) together with the boundary conditions (7) and (8). This problem is solved numerically in the next section.

\section{Numerical results}

We solve (6) with the boundary conditions (7) and (8) by finite differences. We first define a new variable $t$ by the relation

$$
\psi=-t^{n} \text {. }
$$

The parameter $n$ in (9) is used to concentrate mesh points near $\psi=0$. Most of the calculations were performed with $n=2$. The derivatives with respect to $\psi$ in (6), (7) and (8) are given in terms of derivatives with respect to $t$ by the relations

$$
\begin{gathered}
\frac{\partial}{\partial \psi}=-\frac{1}{n} t^{1-n} \frac{\partial}{\partial t} \\
\frac{\partial^{2}}{\partial \psi^{2}}=\frac{1}{n}\left(\frac{1}{n}-1\right) t^{1-2 n} \frac{\partial}{\partial t}+\frac{1}{n^{2}} t^{2-2 n} \frac{\partial^{2}}{\partial t^{2}} .
\end{gathered}
$$

Next we introduce the mesh points $\left(\phi_{I},-t_{J}^{n}\right), I=1, \ldots, M, J=1, \ldots, N$. Here

$$
\begin{gathered}
\phi_{I}=-A h+(I-1) h, \\
t_{J}=\frac{J-1}{N-1} .
\end{gathered}
$$

We satisfy (6) at the mesh points $\left(\phi_{I},-t_{J}^{n}\right), I=2, \ldots, M-1, J=$ $2, \ldots, N-1$. The derivatives are evaluated by central differences. This leads 

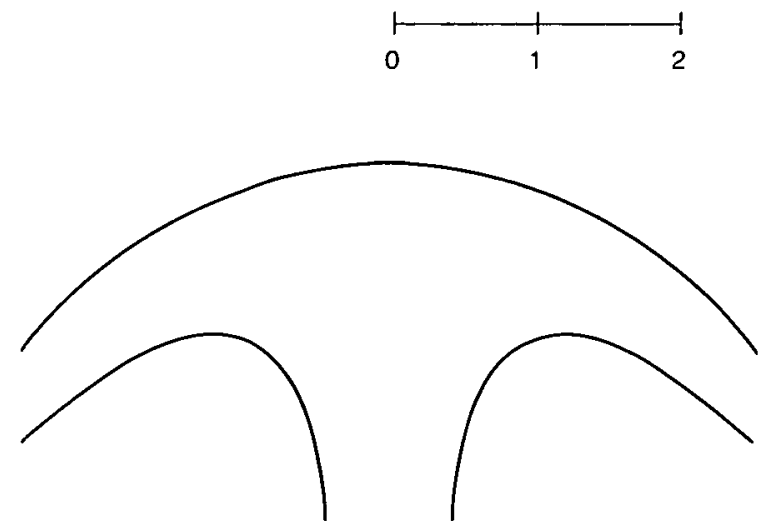

Figure 4. Computed free surface profiles of a two-dimensional jet aimed vertically upwards. The broken line corresponds to the asymptotic solution (22).

to $M N-2 N-2 M+4$ equations for the $M N$ unknowns $y\left(\phi_{I},-t_{J}^{n}\right) .2 M-$ $A-5$ equations are obtained by satisfying (8) at $t_{N}, \psi_{I}, I=2, \ldots, M-1$, and at $t_{1}, \phi_{I}, I=A+2, \ldots, M-2 . A-1$ equations are derived by satisfying (7) at $I=2, \ldots, A$. $N$ equations express the values of $y$ at $\left(\phi_{M},-t_{J}^{n}\right), J=1, \ldots, N$, in terms of the values of $y$ at $\left(\phi_{M-1},-t_{J}^{n}\right)$ and $\left(\phi_{M-2},-t_{J}^{n}\right), J=1, \ldots, N$, by a two-point linear extrapolation formula.

The value of $y$ at $t_{1}, \phi_{A+1}$ is also expressed by in terms of the value of $y$ at $t_{1}, \phi_{A+2}$ and at $t_{1}, \phi_{A+3}$ by a two-point extrapolation formula. Similarly, the value of $y$ at $t_{N}, \phi_{1}$ is related to the values of $y$ at $t_{N}, \phi_{2}$ and $t_{N}$, $\phi_{3}$. An extra equation is found by imposing $y=0$ at $t_{1}, \phi_{A+1}$. Finally, the last $N-1$ equations are obtained by equating the values of $y$ at $\phi_{1}$, $t_{J}, J=1, \ldots, N-1$, to the value of $y$ at $\phi_{1}, t_{N}$. This leads a system of $M N$ nonlinear algebraic equations for the $M N$ unknowns $y\left(\phi_{I},-t_{J}^{n}\right)$. This system is solved by Newton's method. Once the values of $y$ are obtained, the corresponding values of $x$ are calculated by integrating numerically the identity

$$
\frac{\partial x}{\partial \phi}=\frac{\partial y}{\partial \psi} .
$$

The resulting profiles of the free surfaces are shown in Figure 4. These results were obtained with $h=0.06, N=11, M=54$ and $A=26$. We checked that the results were independent of $h, N, M$ and $A$ within graphical accuracy. 


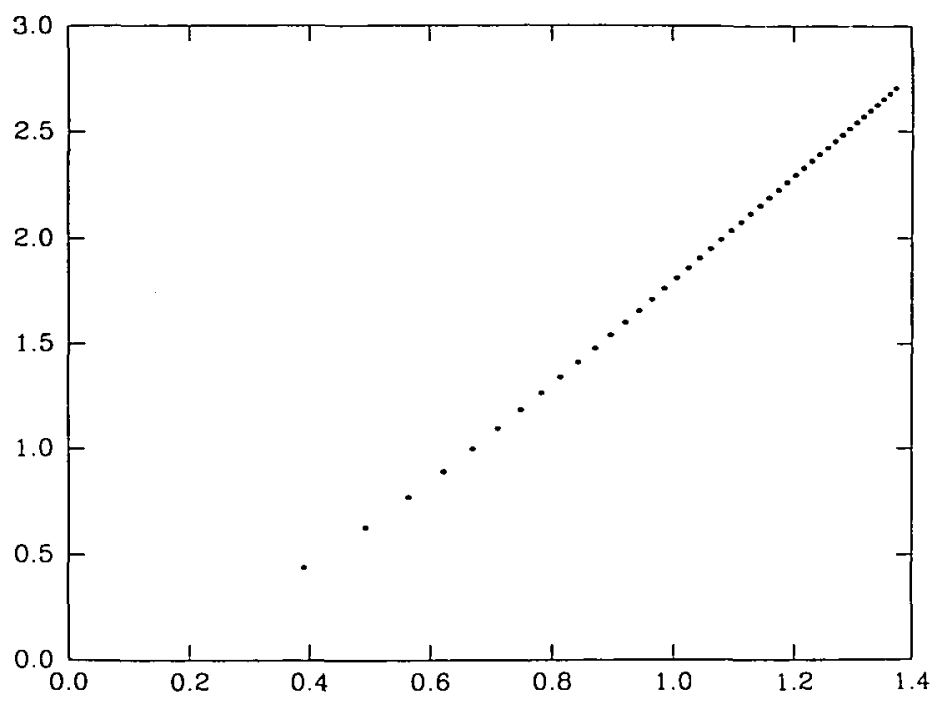

FIGURE 5. Values of $x$ versus $\phi^{1 / 3}$ on the free surface $S B$.

The asymptotic form of the jets as $y \rightarrow-\infty$ can be evaluated by following the analysis of Keller and Geer [4] (see also Tuck and Vanden-Broeck [5]). At $C$, the jet is vertical and

$$
Z=-i a f^{2 / 3}+O(1) \quad \text { as } \phi \rightarrow-\infty .
$$

At $B$, there is a nonzero horizontal velocity and

$$
Z=-i b f^{2 / 3}+d f^{1 / 3}+O(1) \quad \text { as } \phi \rightarrow+\infty .
$$

Here $a, b$ and $d$ are constants.

The constants $a$ and $b$ can be evaluated by substituting (15) and (16) into (8). Retaining only the leading order terms, we obtain after some algebra

$$
a=b=\left(\frac{9}{8}\right)^{1 / 3} \text {. }
$$

In order to evaluate $d$ we set $\psi=0$ in (16) and equate real parts. This leads to

$$
x=d \phi^{1 / 3}+O(1) \quad \text { as } \phi \rightarrow+\infty .
$$

In Figure 5 we present numerical values of $x$ versus $\phi^{1 / 3}$ on the free surface $S B$. The curve is close to a straight line of slope 2.4 for $\phi$ large. Therefore

$$
d \sim 2.4
$$


Finally we derive an explicit equation for the free surface of the jet at $C$. Using conservation of mass we have

$$
\left(\frac{\partial \phi}{\partial y}\right) x=1
$$

on the free surface as $y \rightarrow-\infty$. From (15) we find

$$
\phi \sim\left(\frac{y}{a}\right)^{3 / 2} \text {. }
$$

Differentiating (21) with respect to $y$ and substituting in (20), we obtain

$$
y \sim-\frac{1}{2 x^{2}} .
$$

The relation (22) is represented by the broken line in Figure 4 .

\section{Acknowledgement}

This research was sponsored by the National Science Foundation under Grant No. DMS-8903093.

\section{References}

[1] F. Dias and J.-M. Vanden-Broeck, "Flows emerging from a nozzle and falling under gravity", J. Fluid Mech. 123 (1990) 465.

[2] J.-M. Vanden-Broeck and J. B. Keller, "Weir flows", J. Fluid Mech. 176 (1987) 283.

[3] G. Dagan and M. P. Tulin, "Two-dimensional free-surface gravity flow past blunt bodies", J. Fluid Mech. 51 (1972) 529.

[4] J. B. Keller and J. Geer, "Flows of thin streams with free boundaries", J. Fluid Mech. 59 (1973) 417.

[5] E. O. Tuck and J.-M. Vanden-Broeck, "Splashless bow flows in two dimensions?", 15th Symp. Naval Hydrodynamics, Hamburg, (1985). 